\title{
Novel Experimental Technique for Visualizing and Analyzing Robot- Soil Interactions
}

\author{
K. Skonieczny ${ }^{1}$, S. Moreland ${ }^{2}$, C. Creager $^{3}$, D. Wettergreen ${ }^{4}$ \\ ${ }^{1}$ Carnegie Mellon University, Robotics Institute, 5000 Forbes Ave., Pittsburgh, PA \\ 15213; Ph (412) 268-3856; email: kskoniec@cmu.edu \\ ${ }^{2}$ Carnegie Mellon University, Robotics Institute, 5000 Forbes Ave., Pittsburgh, PA \\ 15213; Ph (412) 268-9576; email: smoreland@cmu.edu \\ ${ }^{3}$ NASA Glenn Research Center, Mail Stop 23-3, 21000 Brookpark Road, Cleveland, \\ OH 44135; Ph (216) 433-6303; email: colin.m.creager@nasa.gov \\ ${ }^{4}$ Carnegie Mellon University, Robotics Institute, 5000 Forbes Ave., Pittsburgh, PA \\ 15213; Ph (412) 268-9576; email: dsw @ ri.cmu.edu
}

\begin{abstract}
A novel experimentation and analysis technique has been developed to enable detailed investigation of robot interactions with granular regolith. This technique, the Shear Interface Imaging Analysis Tool, provides visualization and analysis capability of soil shearing and flow as it is influenced by a wheel or excavation tool. The method places an implement (wheel, excavation bucket, etc.) in granular soil up against a transparent sidewall. During controlled motion of the implement, images are taken of the sub-surface soil, and are processed with optical flow software. Analysis of the resulting displacement field identifies clusters of soil motion and shear interfaces. The Shear Interface Imaging Analysis Tool enables analysis of robot-soil interactions in richer detail than possible before. Prior art relied on longexposure images that provided only qualitative insight, while the new processing technique identifies sub-millimeter gradations in motion and can do so even for high frequency changes in motion (several Hz). Results are presented for various wheel types and locomotion modes: small/large diameter, rigid/compliant, with/without grousers, and rolling/inching. Results are also presented for an excavation bucket horizontally cutting granular soil.
\end{abstract}

\section{INTRODUCTION}

Sustained space exploration requires robots and vehicles that can purposefully interact with granular regolith, such as that covering the surfaces of the Moon, Mars, and other planetary bodies. Traversing great distances is much easier with robust and efficient wheels, and constructing infrastructure requires tools that excavate. Techniques that provide a deeper understanding of how wheels and excavation tools interact with soil will facilitate effective designs and operations.

Observing subsurface soil motion near the robot-soil interface provides information about how soil is interacting with wheels and tools that cannot be gleaned from observing the soil surface. In nominal operation, it is not possible to look through soil at subsurface particles. However, experiments with implements up 
against a glass-sided soil bin can provide insights into interactions at various conditions.

Direct observation of soil motion through glass sidewalls has been utilized in soil mechanics and terramechanics research for over half a century [Bekker 1948]. Wong concluded experimentally that as long as shear stress between glass and soil is negligible, the glass surface acts as a plane of symmetry and the soil behaves as it would directly below an implement twice as wide [Wong 1966, Wong 1967].

One archetypical photographic method for observing soil motion uses longexposure photos and distinguishes sharp and streaking soil grains as stationary and moving, respectively. Streaks in the photos also provide information about the directions of soil motion. With advances in digital camera technology and computer vision processing techniques, new methods providing much richer data have become possible [Gachet 2003, White 2003]. The image processing technique discussed in this work is similar in its implementation to Murthy's, who studied sand displacement under a footing-like indenter [Murthy 2009]. This work presents the Shear Interface Imaging Analysis Tool, which applies a type of these newest soil observation techniques to wheel and implement interactions pertinent for planetary robots.

Previous work demonstrated the Shear Interface Imaging Analysis Tool's utility in determining how soil failure under wheels is modified by implementing a novel inching locomotion mode [Moreland 2011a] as well as how soil displaces under wheels of varying diameter and compliance [Moreland 2011b]. This paper briefly reviews these results and also presents new findings for wheels with grousers as well as excavation buckets.

\section{EXPERIMENTAL AND PROCESSING TECHNIQUE}

The experimental apparatus consists of a glass-walled soil bin filled with relevant regolith simulant, an implement (wheel, excavation bucket, etc.), an actuated horizontal axis of motion and a high-speed camera (Figure 1). When the implement module is equipped with a wheel (Figure 2), its rotations are position or velocity controlled in coordination with the horizontal axis to create a commanded, constant slip as the wheel travels forward. A linear rail allows the wheel to translate freely in the vertical direction allowing for natural sinkage to occur. This also allows for the transmission of a deadweight normal payload to be applied to the wheel. When the implement module is equipped with an excavation bucket, the vertical rail is locked and the rotary actuator is removed.

A 6-d.o.f. force/torque sensor is incorporated to measure the reaction loads in all directions. Sinkage is also measured via an optical encoder affixed to the vertical free linear axis. All telemetry; wheel angular velocity, travel velocity, slip, sinkage, load and power are logged simultaneously at $20 \mathrm{~Hz}$ or higher. 


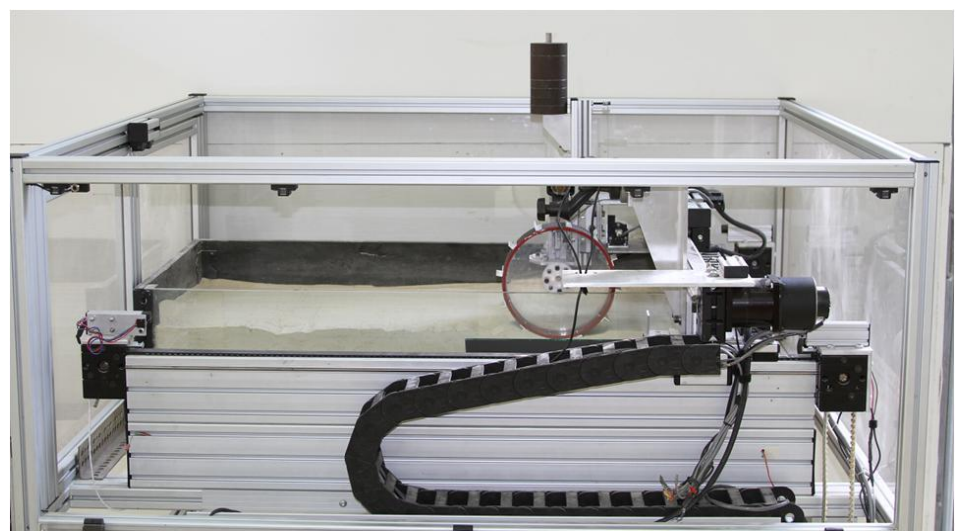

Figure 1. Glass-walled soil bin with horizontal axis of motion.

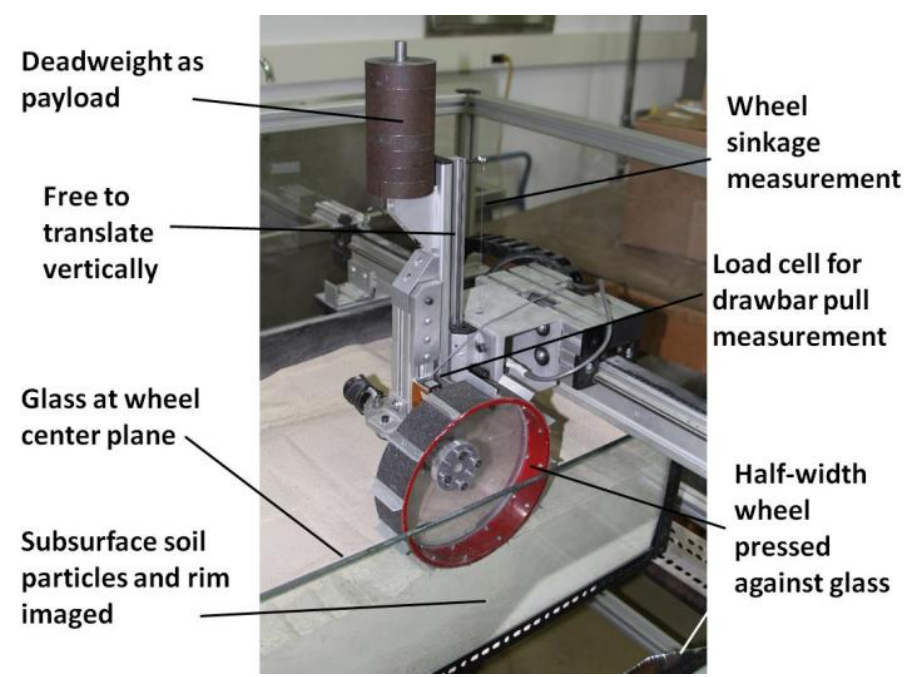

Figure 2. Implement module equipped with wheel.

The test implement is pressed against a sheet of tempered glass that extends the depth of the soil bin. The implement is of half the width of the actual specimen of interest, and correspondingly half the payload weight is applied. This setup simulates a full implement in unconstrained conditions with the same ground pressure, assuming a plane of symmetry at its centerline. Low shearing resistance between the implement and glass boundary is achieved by using tempered glass with a high hardness surface and by the low pressure of the soil particles against the glass wall.

A digital SLR camera with a $50 \mathrm{~mm}$ macro lens is used to image the soil where it interfaces with the test implement, logging frames simultaneously with the rest of the telemetry. A frame rate of 8 frames-per-second is used and is sufficiently fast for the slow implement speeds. The camera is mounted perpendicular to the soil bin glass wall and travels with the implement in the horizontal direction as the carriage moves. External halogen flood lights at a high angle (from the normal) to the glass illuminate the soil particles.

Image processing comprises of optical flow and clustering techniques. An overview of the process described herein is presented in Figure 3. The optical flow algorithm [Black 1996] tracks displacement of soil regions relative to a prior frame and calculates a motion vector at each pixel. Initial clustering separates each image 
into "soil" and "not soil" regions. Additional processing and output is continued only for "soil" regions. The magnitude of flow at each pixel of the soil regions is calculated from the optical flow vector fields. Soil flow is clustered into "significant" and "insignificant" magnitudes of motion. No explicit threshold is used to demarcate these clusters, but rather automatically adaptive $k$-means clustering is used. The shear interface is derived from the boundary between significant and insignificant motions. Soil flow direction is calculated from the optical flow vector fields, for soil regions exhibiting significant soil flow. Soil flow in any direction (360 degrees) is visualized, and an additional boundary is identified at points where the soil transitions between forward and rear flow. Figure 4 is a sample output of the process, showing soil flow magnitude, shear interface between significant and insignificant flow, soil flow direction (within region of significant flow), and boundary between forward and rear flow.

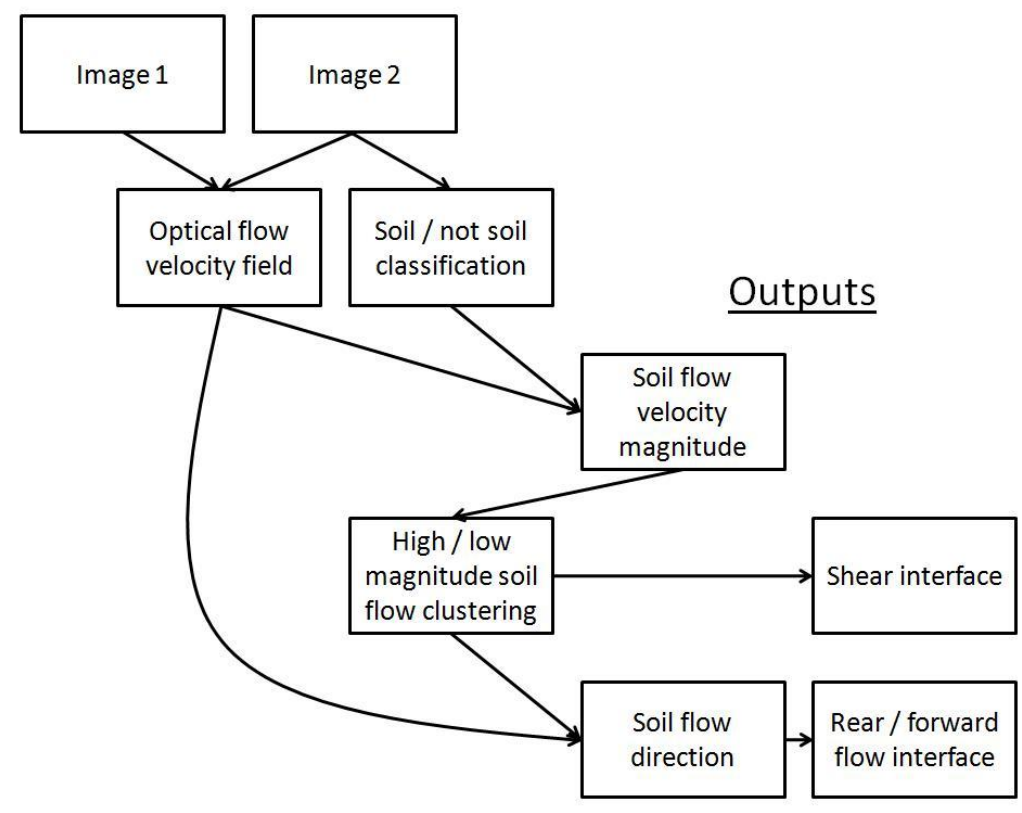

Figure 3. Overview of image processing and output. 


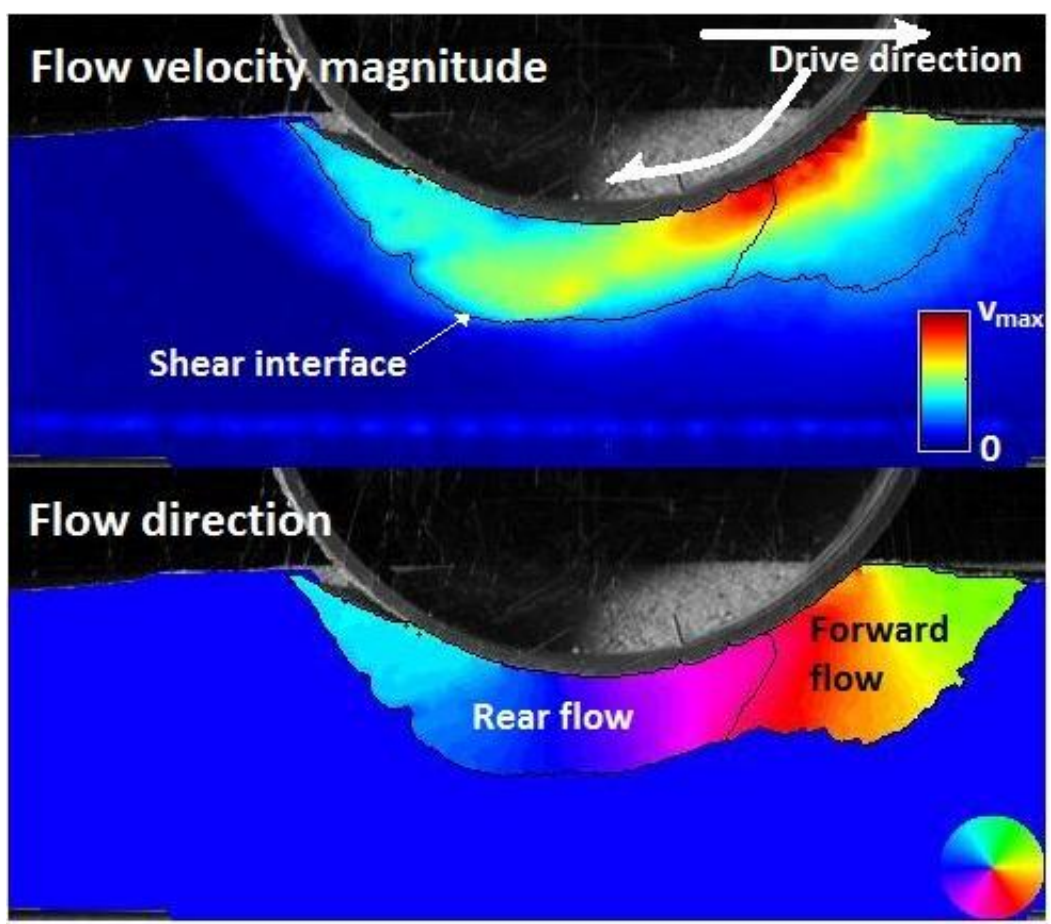

Figure 4. Sample processed output for driven wheel. Soil flow speed (upper) is denoted from blue (static) to red (max. speed). Soil flow direction (lower) within the shear interface is denoted according to the color wheel is the bottom right.

\section{SHEAR IMAGING RESULTS AND DISCUSSION}

Small and large diameter wheels. Large diameter wheels disturb soil less than smaller diameter wheels. Figure 5 shows flow magnitude and flow direction plots for 16 " diameter and 9" diameter wheels. The wheels both have a width of 2.25 ", and both are run at $20 \%$ slip. Images and flow magnitudes are shown to the same scale for direct comparison. The small diameter wheel shears a larger volume of soil, and the resulting flow is of a higher magnitude than under a large diameter wheel. The changes in direction of flow under a small wheel are also much more pronounced than under a large wheel; soil under a small wheel moves both down and then back up more.

Equal normal payload is applied to the large and small diameter wheels. From the images one can see that contact length is not substantially different, meaning that average ground pressure is similar in the two cases. The differences in shearing appear to not be caused by a difference in ground pressure, but rather by the curvature of the wheel rim. The path of soil sheared by a wheel rim follows the shape of the rim itself. A smaller diameter wheel has greater rim curvature, causing sheared soil to experience much more vertical motion. This vertical compaction of the soil is an important source of rolling resistance. 


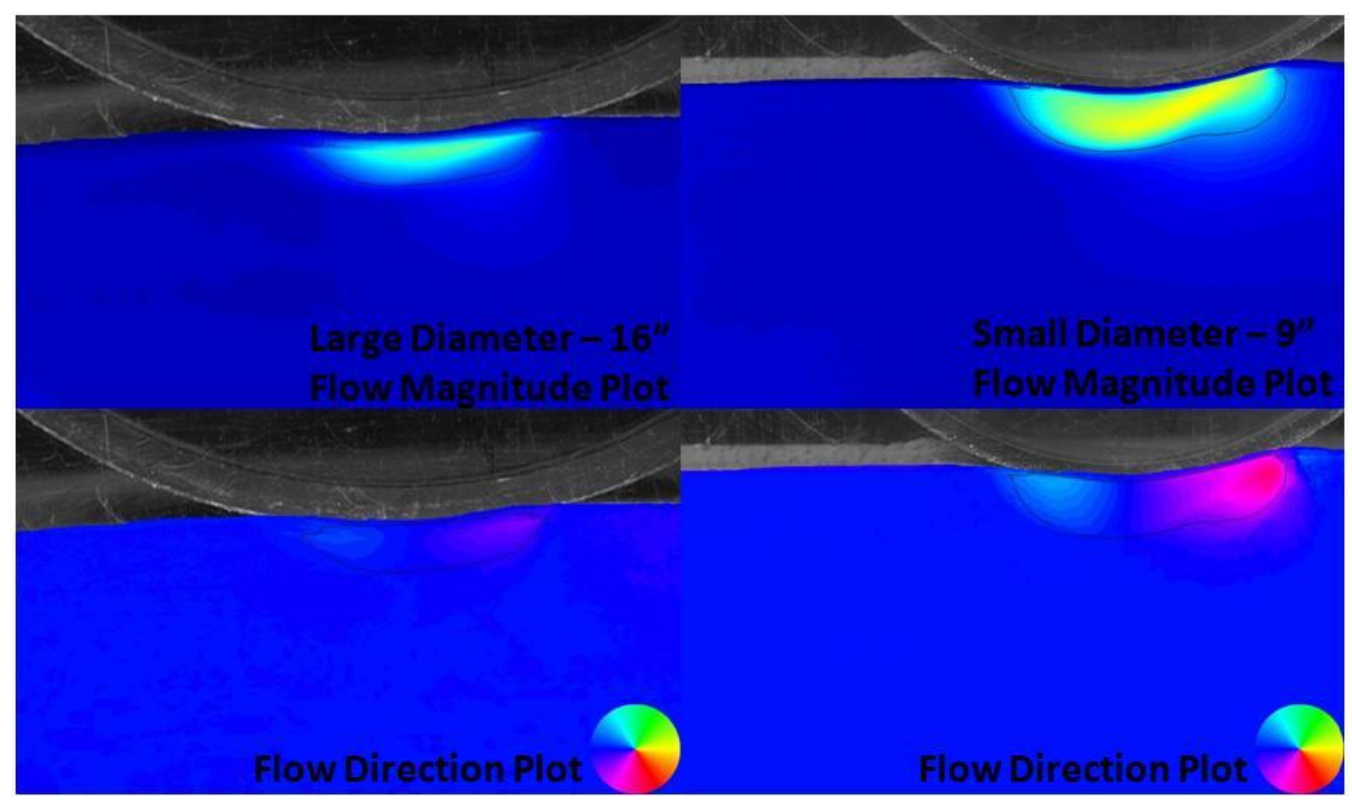

Figure 5. Large diameter wheels (left) disturb soil less than small wheels (right).

Compliant wheels. Compliant wheels disturb soil much less than rigid wheels of any diameter because of their ability to increase footprint size and conform to the terrain. Figure 6 shows shear imaging results, flow magnitude and direction, for a compliant wheel driving with $20 \%$ slip. The magnitude scale is greatly magnified relative to that used in the previous section for large and small diameter rigid wheels; at the same scale, the flow magnitude for the compliant wheel is barely distinguishable from static soil. The direction plot shows that soil is flowing predominantly horizontally, with only a small downward component near the front of the wheel.

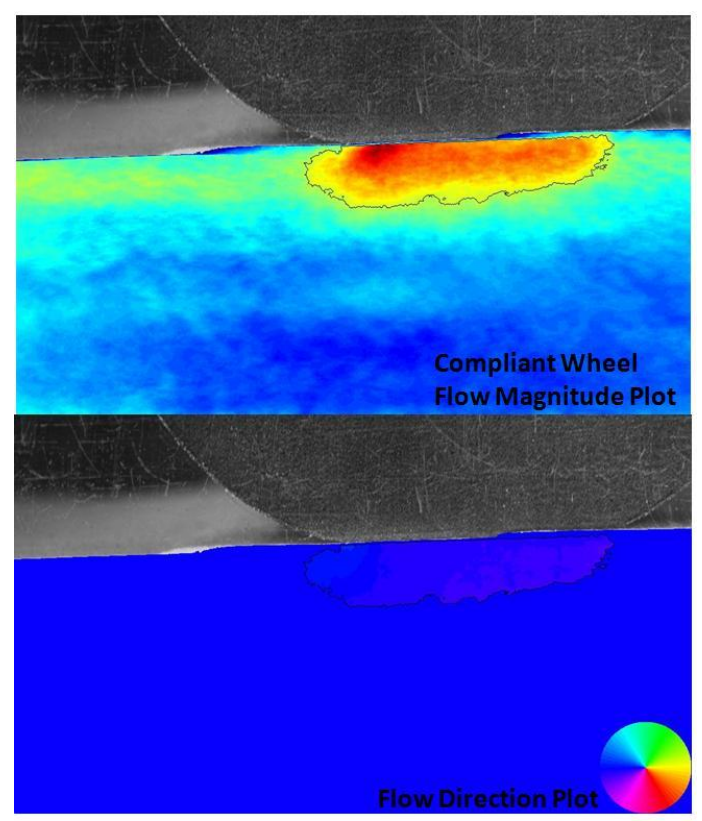

Figure 6. A compliant wheel exhibits low magnitude, nearly horizontal shearing. 
The low magnitude and nearly horizontal shearing of compliant wheels can be seen as a continuation of the changes observed when increasing from smaller to larger diameter wheels. Sufficiently compliant wheels take on the shape of the flat terrain they traverse, resulting in a contact patch with zero curvature, analogous to a wheel of infinite diameter.

Reducing disturbance of traversed soil can result in lower soil resistance, lower sinkage, and lower risk of entrapment. Larger diameter and compliant wheels ought to be considered for planetary applications because of these advantages.

Wheels with grousers. Grousers are often utilized to increase performance of rover wheels. A wheel's net traction (drawbar pull) can be increased by increasing thrust, decreasing resistance, or both. The Shear Interface Imaging Analysis Tool shows that decreased resistance is an important factor in the increased performance of wheels with grousers.

Figure 7 shows soil flow beneath wheels without and with grousers. The tests use the same wheel and payload, but the wheel on the right has sixteen $1 / 2$ " aluminum grousers. Flow magnitudes and directions are averaged over 50 frames. Flow magnitude is saturated to display the low end of magnitude values.
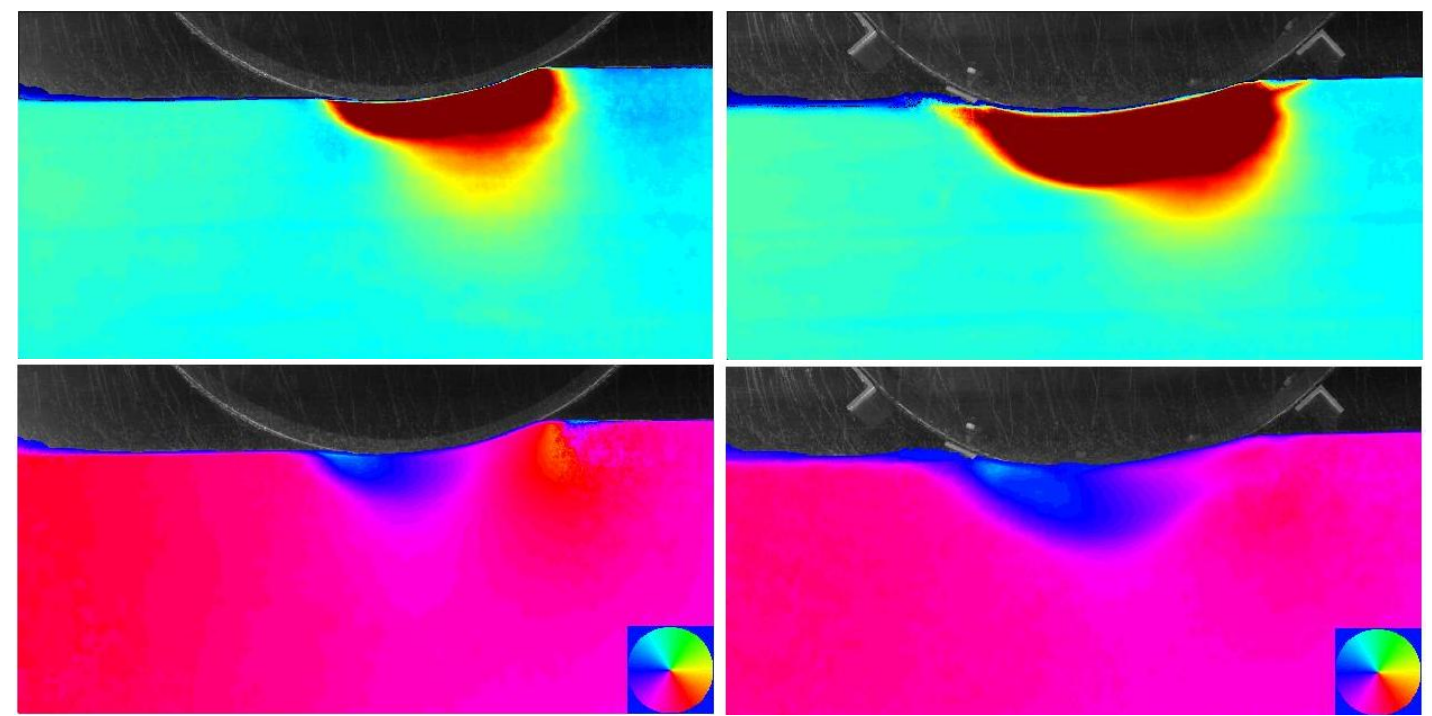

Figure 7. Grousers reduce forward flow in soil at the fore of a wheel. Magnitude plots (upper) are saturated to display low end of magnitude values.

The flow direction plot for the grouser-less wheel shows a region of soil at the fore of the wheel-soil contact that has a significant forward component (orange color). The flow magnitude in this region is low, but nonetheless soil is being compacted forward. Forward compaction of soil ("bull-dozing") can only contribute resistance to forward wheel motion. A wheel with grousers shows no comparable region of forward flow; the grousers appear to significantly reduce the bull-dozing effect. In regions of comparably low flow magnitude, soil flow direction is indistinguishable from the surrounding bulk soil. 
When a grouser interacts with the ground at the fore of wheel-soil contact, its geometry and motion confine soil and prevent it from being pushed forward. Lack of forward soil flow results in lower resistance, and hence higher drawbar pull performance.

Inching locomotion. Rovers with reconfigurable suspensions and wheelbases are able to perform novel locomotion modes. One such locomotion mode, inching, involves driving a subset of a robot's wheels forward while keeping the others static relative to the ground. The subset of driven wheels is cycled, resulting in forward motion of all the wheels and hence the robot itself. Inching has shown significant increases in drawbar pull performance over nominal driving at high loads [Moreland 2011a].

Drawbar pull increases with decreased resistance, increased thrust, or both. Wheels kept static relative to the ground do not contribute any rolling resistance, so this contributes to an increase in drawbar, though only by a small amount.

Figure 8 shows soil shearing beneath a rolling and a statically pushed wheel. From the shape of the shear interfaces, it is clear that different mechanisms are employed to produce thrust in the two cases. In the pushed wheel case, soil moves more as a block, similar to ground failure beneath a loaded plate or foundation. The difference in shearing mechanism enables higher thrust loads to be produced with the pushed wheel.
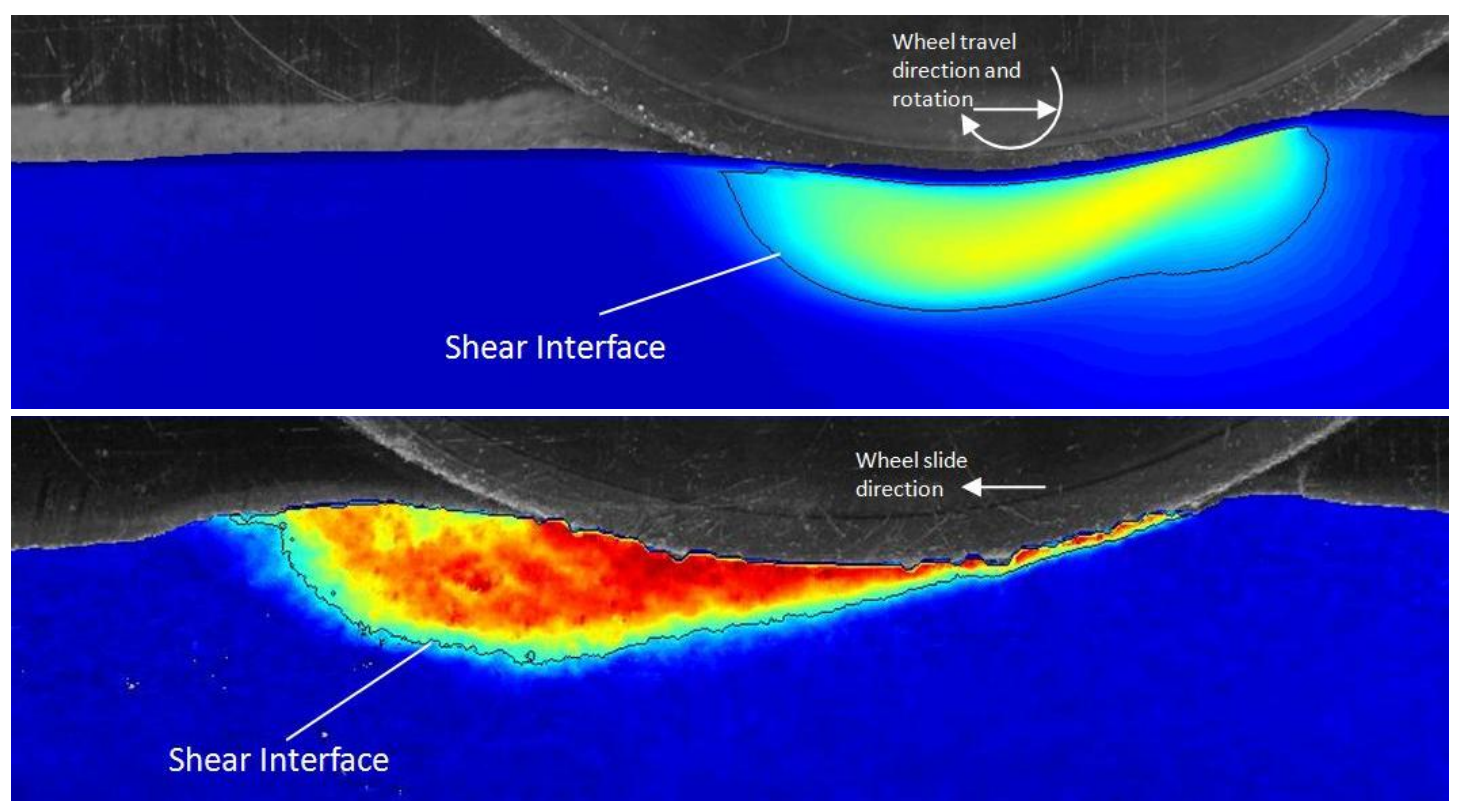

Figure 8. Soil shearing producing different thrust mechanisms for a driven wheel (upper) and static pushed wheel (lower).

Excavation bucket. Tools for excavation are another important regime, besides wheels, for robot-soil interactions. Agui describes the evolution of a pile of lunar simulant at the leading edge of a bucket cutting horizontally, based on measurements of the soil surface [Agui 2010]. Figure 9 shows how the Shear Interface Imaging Analysis Tool images motion below the soil surface. The bucket in this analysis has 
proportional (half-scale) dimensions as that described by Agui, and is commanded at equally half-scaled depth and forward speed, and the same cut angle.

All soil in the pile is pushed forward with the bucket (to the left in the image). Soil at the fore of the pile is also pushed up, while soil near the rear of the pile has a downward component to its flow. This analysis demonstrates the feasibility of using an imaging technique to study excavated soil, providing another promising direction for future work.

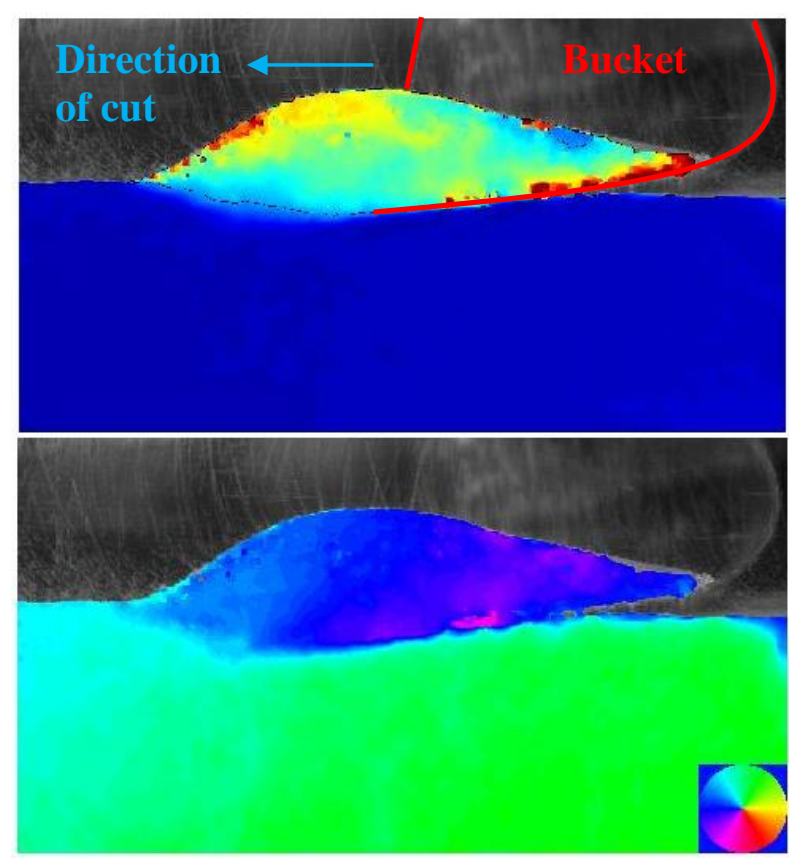

Figure 9. Bucket excavation of soil shows how pile is forming at leading edge

\section{CONCLUSION}

The Shear Interface Imaging Analysis Tool enables analysis of robot-soil interactions in richer detail than possible before. This processing technique identifies submillimeter gradations in motion for a full image-sized flow field.

Analyzing robot-soil interactions at this level of detail enables direct observation of phenomena that have not been previously described based on observations of the soil surface or prior art in subsurface imaging: lack of forward flow at the fore of grousered wheels, alternate thrust generation mechanisms produced during inching, scale and direction of soil disturbance under wheels of varying diameter and compliance, and formation of soil piles in an excavation bucket.

General application of the Shear Interface Imaging Analysis Tool can greatly enhance understanding of processes where robots interact with granular soils, such as driving and digging. 


\section{REFERENCES}

Agui, J. H., and Wilkinson, R. A. (2010). "Granular flow and dynamics of lunar simulants in excavating implements." Earth and Space 2010, ASCE, Honolulu, 84-94.

Bekker, M. G. (1948). "Photographic method of determining the soil action beneath footings." Proc. 2nd Int. Conf. on Soil Mechanics and Foundation Engineering, Rotterdam, 193-194.

Black, M. J., and Anandan, P. (1996). "The robust estimation of multiple motions: Parametric and piecewise-smooth flow fields." CVIU, 63(1), 75-104.

Gachet, P., Klubertanz, G., Vulliet, L., and Laloui, L. (2003). "Interfacial behavior of unsaturated soil with small-scale models and use of image processing techniques." Geotechnical Testing Journal, 26(1).

Moreland, S. J., Skonieczny, K., and Wettergreen, D. S. (2011). "Inching locomotion for planetary rover mobility." IEEE Aerospace, Big Sky.

Moreland, S. J., Skonieczny, K., Wettergreen, D. S., Creager, C., and Asnani, V. (2011). "Soil motion analysis system for examining wheel-soil shearing." Proc. $17^{\text {th }}$ Int. Conf. of the International Society for Terrain-Vehicle Systems, Blacksburg.

Murthy, T. G., Gnanamanickam, E. P. Saldana, C., and Chandrasekar, S. (2009). "Deformation field in indentation of granular materials." Proc. 6th Int. Conf. on Micromechanics of Granular Media, Golden, 263-266.

White, D. J., Take, W. A, and Bolton, M. D. (2003). "Soil deformation measurement using particle image velocimetry (PIV) and photogrammetry." Geotechnique, 53, 619-631.

Wong, J. Y., and Reece, A. R. (1966). "Soil failure beneath rigid wheels." Proc. $2^{\text {nd }}$ Int. Conf. of the International Society for Terrain-Vehicle Systems, Quebec City, 425-445.

Wong, J. Y. (1967). "Behaviour of soil beneath rigid wheels." J. Agric. Engng Res., 12(4), 257-269. 